\title{
Layer-by-layer thermal conductivities of the Group III nitride films in blue/green light emitting diodes
}

\author{
Zonghui Su, ${ }^{1}$ Li Huang, ${ }^{2}$ Fang Liu, ${ }^{2}$ Justin P. Freedman, ${ }^{2}$ Lisa M. Porter, ${ }^{2}$ Robert F. Davis, ${ }^{2}$ \\ and Jonathan A. Malen ${ }^{1, a)}$ \\ ${ }^{1}$ Department of Mechanical Engineering, Carnegie Mellon University, Pittsburgh, Pennsylvania 15213, USA \\ ${ }^{2}$ Department of Materials Science and Engineering, Carnegie Mellon University, Pittsburgh, Pennsylvania \\ 15213, USA
}

(Received 14 January 2012; accepted 30 April 2012; published online 16 May 2012)

\begin{abstract}
Thermal conductivities $(k)$ of the individual layers of a GaN-based light emitting diode (LED) were measured along [0001] using the 3-omega method from 100-400 K. Base layers of AlN, GaN, and InGaN, grown by organometallic vapor phase epitaxy on $\mathrm{SiC}$, have effective $k$ much lower than bulk values. The $100 \mathrm{~nm}$ thick AlN layer has $k=0.93 \pm 0.16 \mathrm{~W} / \mathrm{mK}$ at $300 \mathrm{~K}$, which is suppressed $>100$ times relative to bulk AlN. Transmission electron microscope images revealed high dislocation densities $\left(4 \times 10^{10} \mathrm{~cm}^{-2}\right)$ within AlN and a severely defective AlN-SiC interface that cause additional phonon scattering. Resultant thermal resistances degrade LED performance and lifetime making layer-by-layer $k$, a critical design metric for LEDs. (C) 2012 American Institute of Physics. [http://dx.doi.org/10.1063/1.4718354]
\end{abstract}

More than $20 \%$ of electricity in the United States is consumed by lighting. Solid-state light emitting diodes (LEDs) hold considerable potential to be more efficient and effective sources of artificial light than incandescent and fluorescent technologies. ${ }^{1}$ Group III nitride-based blue and green LEDs are currently being developed for this purpose. Device architectures consist of light-emitting multiple quantum wells (MQWs) supported by base layers of aluminum nitride (AlN), gallium nitride $(\mathrm{GaN})$, and indium gallium nitride (InGaN). The MQW itself is a periodic structure composed of alternating InGaN wells and $\mathrm{GaN}$ barriers.

Heat generation and removal in the LED are complicated by multiple interfaces, causing high operating temperatures that degrade efficiency, shift the emission spectrum, and reduce the lifetime of LEDs. ${ }^{2}$ Prior experimental investigations of bulk GaN and AlN have shown that thermal transport is phonon-dominated. ${ }^{3-6}$ Previous thin film studies have been (i) based on ideal films with low defect concentrations that are unrepresentative of LED grade films $\mathrm{s}^{3,7}$ or (ii) focused on high electron mobility transistors (HEMTs), rather than LED architectures. ${ }^{8-10}$ Neither submicron GaN films nor real nitride based LED devices have been investigated.

Studies of Si and other thin films have demonstrated that thermal conductivity is greatly reduced when the thickness of the film or the defect spacing is less than the intrinsic mean free path of the phonons., ${ }^{5,11}$ Nitride based LED structures are fabricated from multiple layers of thin films ranging from $3 \mathrm{~nm}$ to $100 \mathrm{~nm}$ in thickness; whereas, the spectrum-average phonon mean free path in $\mathrm{GaN}$ at $300 \mathrm{~K}$ is $\sim 100 \mathrm{~nm},{ }^{13}$ and the longest phonon mean free paths in the spectrum are much greater. ${ }^{14}$ To achieve economic viability, the nitride films are grown by organometallic vapor phase epitaxy (OMVPE) on foreign substrates, including SiC, sapphire, and Si. Mismatched lattice constants at the film-

${ }^{\text {a)} E m a i l ~ a d d r e s s: ~ j o n m a l e n @ a n d r e w . c m u . e d u . ~}$ substrate interface create high densities of atomic and line defects, the latter of which that extend throughout the device as a source of phonon scattering. Given these considerations, this paper reports layer-by-layer experimental measurements of thermal conductivity in real nitride based LED architectures grown on [0001]-oriented SiC substrates. The OMVPE growth process involves the activated chemisorption of precursor gases on the surface of a heated substrate. The thickness and composition of the films are controlled by the ratio of precursors, their flow rates, and the substrate temperature. $\mathrm{GaN}$ cannot be directly grown on SiC (Refs. 15-18) at the normal deposition temperatures used to produce nitridebased LED structures. Instead, a nucleation layer must be grown that is sufficiently closely matched in chemistry, crystallography, lattice parameters, and the coefficients of thermal expansion with the substrate and the subsequently grown layer. For $\mathrm{SiC}$, the most appropriate nucleation layer is AlN. In the research described, herein, an AlN nucleation layer $(100 \mathrm{~nm})$ followed by a GaN buffer layer $(100 \mathrm{~nm})$ and a graded $\mathrm{GaN}-\mathrm{In}_{10} \mathrm{Ga}_{90} \mathrm{~N}$ layer $(90 \mathrm{~nm})$ formed the base for the six and a half repeated units of $(\mathrm{GaN})_{8 \mathrm{~nm}}$ barriers and $\left(\mathrm{In}_{20} \mathrm{Ga}_{80} \mathrm{~N}\right)_{3 \mathrm{~nm}}$ wells of the MQW stacks (see Fig. 1).

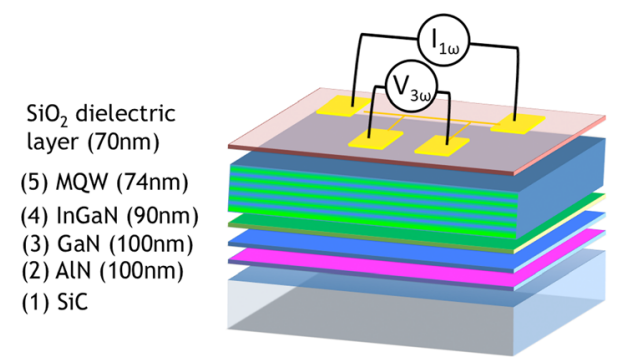

FIG. 1. Representative sample structures of green-emitting nitride-based LED devices (figure not drawn to scale). The five samples are as follows: $(1),(1)+(2),(1)+(2)+(3),(1)+(2)+(3)+(4)$, and $(1)+(2)+(3)+(4)+(5)$. The $\mathrm{SiO}_{2}$ dielectric layer was added to each sample. The 3-omega gold pattern is enlarged in the figure. The gold line between pads performs as both a heater and a thermometer to extract the film thermal conductivities. 
All films were deposited in a vertical, resistively heated, cold wall, pancake-style system. Each $\mathrm{SiC}$ substrate was ultrasonically cleaned in solvents, submersed in a 10:90 $\mathrm{HF}: \mathrm{H}_{2} \mathrm{O}$ solution to remove the native oxide and heated in flowing $\mathrm{H}_{2}$ to $1100^{\circ} \mathrm{C}$ within the pre-evacuated $\left(10^{-6} \mathrm{Torr}\right)$ deposition chamber to initiate the growth of the AlN. The reactants of trimethylaluminum and triethylgallium (both carried in $\mathrm{H}_{2}$ ) and trimethyindium (carried in $\mathrm{N}_{2}$ ) and ammonia were premixed at the entrance of the deposition chamber and subsequently combined with either a $\mathrm{N}_{2}$ or a $\mathrm{H}_{2}$ diluent for growth of the MQW structures and the underlying films, respectively. The temperatures of 1100,1000 , and $790^{\circ} \mathrm{C}$ and total pressures of 20 Torr, 20 Torr, and 100 Torr were used for growths of AlN, GaN, and the MQWs, respectively. Five samples were grown with each sample having one additional layer (mechanically polished $\mathrm{SiC}$ substrate, $\mathrm{AlN}, \mathrm{GaN}$, $\mathrm{InGaN}$, and $\mathrm{MQW}$ ) compared with the previous sample.

The 3-omega method was used to measure the layer-bylayer thermal conductivities of the LED structure based on its ability to accurately measure thermal conductivity in thin films. ${ }^{19,20}$ A differential approach described by Lee and Cahill ${ }^{20}$ was used, where the 3-omega signal of one sample was subtracted from the signal of the sample with just one more layer to isolate the thermal properties of each thin film as labeled Eqs. (2)-(5) in Figure 1. Gold 3-omega heating lines and wire-bonding pads were microfabricated on all samples using photolithography. Before patterning, a $70 \mathrm{~nm}$ dielectric layer of $\mathrm{SiO}_{2}$ (Ref. 21) was sputtered onto the LED samples to electrically isolate the 3-omega heater from the semi-conductor films. ${ }^{20}$ The in-plane width and length of the heating lines were $12 \mu \mathrm{m}$ and $1500 \mu \mathrm{m}$, respectively.

Temperature dependent experiments were performed within a cryostat. 3-omega experimental data were fit using an analytical heat conduction model to extract the unknown thermal conductivities of individual films within the stacked LED structure. ${ }^{20,22}$ The thicknesses of the individual nitride films were much less than the width of the heater, therefore the heat flow is one dimensional and related to the thermal conductivity in the [0001] direction. ${ }^{20}$ Under this condition, Lee and Cahill's thin film solution ${ }^{20}$ was valid and fits to the data were made using a least square method. The reported values are effective thermal conductivities, because they include interface thermal resistances. For example, the AlN$\mathrm{SiC}$ interface thermal resistance is wrapped into the reported thermal conductivity of AlN. The thermal conductivities of the GaN, InGaN, MQW, and AlN layers within the LED stacked structure were measured at temperatures ranging from 100 to $400 \mathrm{~K}$, in $50 \mathrm{~K}$ intervals.

The threading dislocation density in GaN and AlN were measured using two different techniques. In the case of GaN, a molten $\mathrm{KOH}$ etch containing $10 \mathrm{wt}$. \% $\mathrm{MgO}$ was used to increase the size of the dislocations. ${ }^{23}$ The enlarged pits were imaged in plan-view with a scanning electron microscope and counted over an area of $\sim 100 \mu \mathrm{m}^{2}$ to determine the dislocation density. The threading dislocation density in AlN was estimated from plan-view transmission electron microscope (TEM) images. The TEM sample was made by mechanical thinning and grinding to a thickness of $30 \mu \mathrm{m}$, followed by ion milling to electron transparency using a Gatan PIPS at $8^{\circ}$ tilt with $\mathrm{Ar}$ ions at $4 \mathrm{keV}$. The plan-view
TEM images were acquired using an FEI Tecnai F20, with the sample viewed along the [0001] axis. The threading dislocation density was averaged over 10 images, representing a total area of $0.6 \mu \mathrm{m}^{2}$.

The results of the thermal conductivity measurements are reported in Table I and Figures 2 and 3. The thicknesses of the individual layers and their effective thermal conductivities at $300 \mathrm{~K}$ are shown in Table I. Uncertainties in predictions of thermal conductivity are based on the propagated uncertainties in the film thicknesses, heater geometry, and heater coefficient of thermal resistance. Temperature dependent thermal conductivities for AlN, GaN, InGaN, and the MQW are plotted in Figures 2 and 3. In general the effective thermal conductivities increased from $100-150 \mathrm{~K}$, and were invariant from $150-400 \mathrm{~K}$. Reductions in the thin film effective thermal conductivities, relative to bulk values, may be attributed to two sources: (1) phonon scattering at closely spaced interfaces and (2) phonon scattering from dislocations within the films. Both of these effects are considered in the discussion that follows.

The GaN layer was found to have the greatest thermal conductivity within the LED stack. Our measurement of $16.0 \mathrm{~W} / \mathrm{mK}$ at $300 \mathrm{~K}$ is an order of magnitude less than that of the bulk GaN crystal, which has been reported to be $\sim 230 \mathrm{~W} / \mathrm{mK}$ at room temperature. ${ }^{24}$ However, it is far higher than the Cahill-Pohl lower limit to thermal conductivity. ${ }^{25}$ SEM images of the GaN revealed a high threading dislocation density of $1.2 \times 10^{8} \mathrm{~cm}^{-2}$, which is equivalent to 1 dislocation every $980 \mathrm{~nm}$. The measurement of polycrystalline $\mathrm{GaN}(\sim 7 \mathrm{~nm}$ grain size) reported by Daly et al. indicates that scattering from grain boundaries results in a thermal conductivity even lower than observed here. ${ }^{26}$

To understand the separate reductions in thermal conductivity due to film boundaries and internal threading dislocations, the truncated Callaway (TC) model detailed in Koh et al. $^{5,27}$ was employed as a predictive tool. Umklapp and normal phonon scattering coefficients $\left(B_{U}\right.$ and $B_{N}$ from Ref. 5) were adjusted to fit data from bulk $\mathrm{GaN},{ }^{28}$ as shown in Figure 2(a). An additional phonon relaxation time was added to the model to account for reductions in the phonon lifetimes due to dislocation scattering. As a simple, yet physical description we assumed that dislocations scatter phonons independent of frequency, and therein limit their mean free paths to the average distance between dislocations $l_{D}$. The relaxation time due to dislocations is hence defined as,

$$
\frac{1}{\tau_{D}}=\frac{v_{g}}{l_{D}}=v_{g} \sqrt{\frac{3^{1 / 2} \rho_{D}}{2}},
$$

TABLE I. Layer-by-layer effective thermal conductivity of Group III nitride LED films at $300 \mathrm{~K}$.

\begin{tabular}{lcc}
\hline \hline Layer & Thickness & $\mathrm{k}_{\text {eff }}$ at $300 \mathrm{~K}(\mathrm{~W} / \mathrm{mK})$ \\
\hline MQW & $74 \mathrm{~nm}$ & $4.3 \pm 0.9$ \\
$\mathrm{InGaN}$ & $90 \mathrm{~nm}$ & $5.2 \pm 1.0$ \\
$\mathrm{GaN}$ & $100 \mathrm{~nm}$ & $16.0 \pm 3.2$ \\
$\mathrm{AlN}$ & $100 \mathrm{~nm}$ & $0.93 \pm 0.16$ \\
$\mathrm{SiC}$ & $250 \mu \mathrm{m}$ & $425 \pm 26$ \\
\hline \hline
\end{tabular}



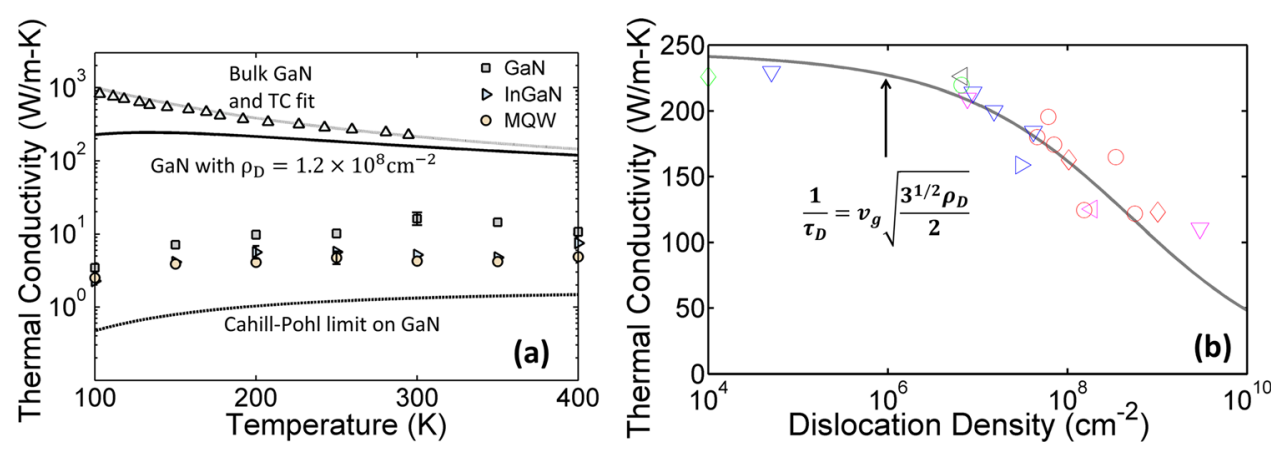

FIG. 2. (a) Temperature dependent data for nitride layers (except AlN) and comparison with experimental values for bulk GaN, as well as TC and Cahill-Pohl theoretical prediction. Representative error bars are shown for one point in each data series. The comparison suggests that the observed reductions in the thermal conductivity of GaN relative to bulk, result from both dislocation density and interface thermal conductance. Alloy scattering and increased interface scattering cause further reductions in the InGaN and MQW films, respectively. (b) Thermal conductivity of GaN vs. dislocation density at $300 \mathrm{~K}$. The new relaxation time based on dislocation density was added to the TC model and compared with data from Mion et al. (see Ref. 24). This revised TC model was used to calculate the solid line in (a) with the measured $\rho_{D}=1.2 \times 10^{8} \mathrm{~cm}^{-2}$ of the GaN film.

where $v_{g}$ is the group velocity, $l_{D}$ is the average distance between dislocations, and $\rho_{D}$ is the areal dislocation density. The factor of $\sqrt{3^{1 / 2} / 2}$ results from the assumption that the dislocations are areally close packed. This additional relaxation time is added to the phonon-phonon relaxation time $\tau_{P-P}$ and defect scattering relaxation time $\tau_{\text {def }}$ by Matthiessen's Rule to create an effective relaxation time,

$$
\frac{1}{\tau_{e f f}}=\frac{1}{\tau_{P-P}}+\frac{1}{\tau_{d e f}}+\frac{1}{\tau_{D}} .
$$

In Figure 2(b) this model's prediction, given the same values of $\tau_{P-P}$ and $\tau_{\text {def }}$ from the bulk GaN calculation in Figure 2(a), is compared to historical room temperature data from Mion et al. for the thermal conductivity of GaN vs. dislocation density. Agreement between the prediction and data verifies that this model captures the effect of dislocations with no additional fitting parameters. We note, as in Mion et al., that earlier models for relaxation time tend to predict a lesser reduction in the thermal conductivity of $\mathrm{GaN}$ due to dislocations. ${ }^{24,29,30}$

The TC Model with the additional $\tau_{D}$ term has been applied to our $\mathrm{GaN}$ film with a dislocation density of
$1.2 \times 10^{8} \mathrm{~cm}^{-2}$. This dislocation density was measured using the $\mathrm{KOH}$ etchant method described earlier. The predicted thermal conductivity, shown as a solid line in Figure 2(a), is lower than the thermal conductivity of a single crystal, yet still offset to higher thermal conductivity than our GaN data. If we assume that the offset between this prediction and our measurement comes from interface thermal resistance at the AlN-GaN interface, we calculate an interface conductance (h) on the order of $150 \mathrm{MW} / \mathrm{m}^{2} \mathrm{~K}$ at $300 \mathrm{~K}$. This value falls within a reasonable range $\mathrm{e}^{31}$ and suggests that both dislocation scattering and interface resistance account for reductions in the effective thermal conductivity of sub-micron GaN films.

The InGaN layer exhibited a lower thermal conductivity than that of the GaN film due to additional alloy scattering. The MQW layer exhibited an even further reduced thermal conductivity compared to the InGaN layer. It is likely that interfaces within the repeated MQW units $\left((\mathrm{GaN})_{8 \mathrm{~nm}} /\right.$ $\left.(\mathrm{InGaN})_{3 \mathrm{~nm}}\right)$, increased phonon scattering. The MQW effective thermal conductivity of $4.3 \mathrm{~W} / \mathrm{mK}$ at $300 \mathrm{~K}$ is similar in magnitude to the value of an AlN-GaN superlattice ${ }^{5}$ reported by Koh et al. $\left(6.35 \mathrm{~W} / \mathrm{mK}\right.$ for $(\mathrm{AlN})_{4 \mathrm{~nm}}-(\mathrm{GaN})_{2.2 \mathrm{~nm}}$ structure).
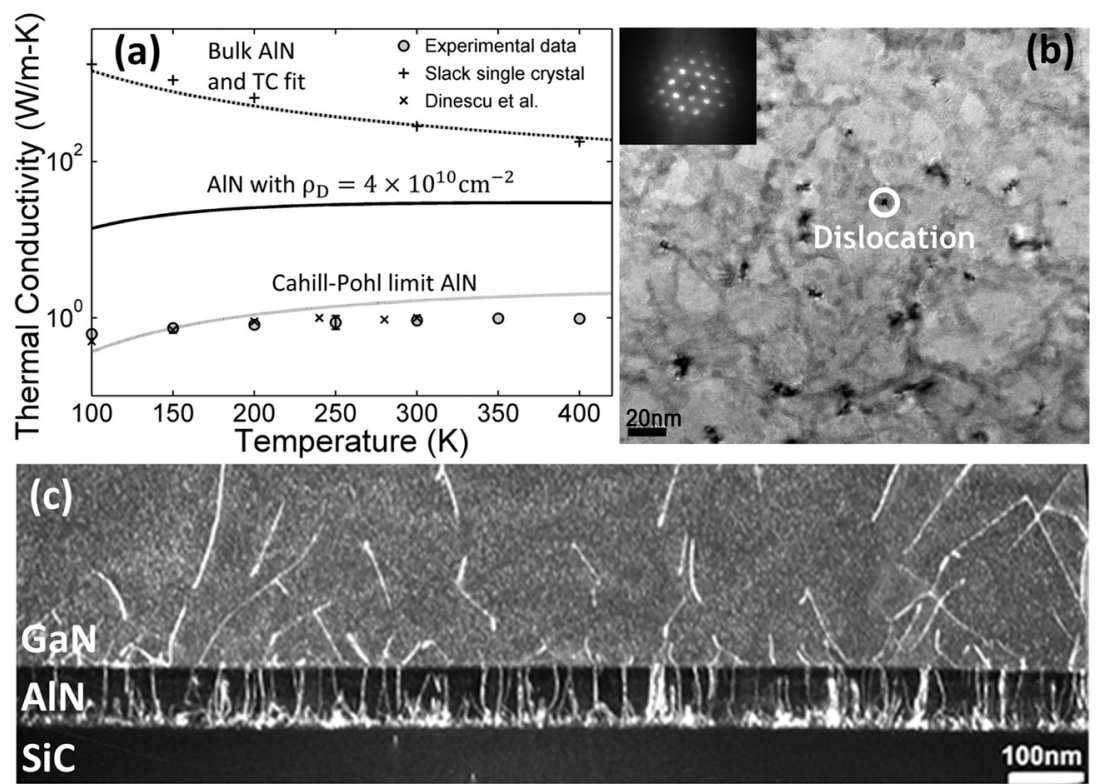

FIG. 3. (a) Temperature dependent thermal conductivity of AlN along [0001] in comparison with single crystal AlN data from Slack (see Ref. 4), pulsed laser deposited AlN data from Dinescu (see Ref. 6), and theoretical predictions from TC and Cahill-Pohl models. (b) A plan view TEM image of the AIN layer acquired along the [0001] zone axis. Inset is the associated diffraction pattern. The TEM image reveals a high dislocation density $4 \times 10^{10} \mathrm{~cm}^{-2}$ that causes additional phonon scattering. Each dark dot in the image represents a dislocation in the AlN film. The solid line in (a) has been calculated using the TC model with $\tau_{D}$ based on $\rho_{D}=4 \times 10^{10} \mathrm{~cm}^{-2}$, and shows that the observed reductions in the thermal conductivity of AlN cannot be solely explained by dislocation scattering. (c) A crosssectional TEM image along [01시 shows increased defect density near the AlN-SiC interface ("Reprinted with permission from Z. J. Reitmeier, S. Einfeldt, R. F. Davis, X. Y. Zhang, X. L. Fang, and S. Mahajan, Acta Mater. 57, 4001 (2009). Copyright (C 2009 Elsevier.”). These defects cause very low interface conductance that further reduces the effective thermal conductivity of the AIN. 
The AlN buffer layer was found to have the smallest thermal conductivity of all the individual layers of interest. Figure 3(a) shows a two order of magnitude reduction in our measured thermal conductivity relative to that of bulk single crystal AlN measured by Slack. ${ }^{4}$ Values of similar magnitude $(1 \mathrm{~W} / \mathrm{mK}$ at $300 \mathrm{~K})$ in AlN films were measured by Dinescu et al. ${ }^{6}$ and Riedel et al. ${ }^{8}$ Dinescu et $a .^{6}{ }^{6}$ also observed temperature invariant thermal conductivity from 77 to $300 \mathrm{~K}$ (reproduced in Figure 3(a)). Plan view TEM images, shown in Figure 3(b), reveal that the AIN layer has an extremely high threading dislocation density, an effect attributed to its lattice mismatch with the $\mathrm{SiC}$ substrate. Based on the TEM images, dislocations that extend through the AlN film have cores that are roughly $54 \mathrm{~nm}$ apart, and thus an areal density of $4 \times 10^{10} \mathrm{~cm}^{-2}$.

Similar to the GaN film analysis, a truncated Callaway model, in agreement with Slack's single crystal values for $\mathrm{AlN},{ }^{4}$ was used to predict the behavior of an AlN film with a dislocation density of $4 \times 10^{10} \mathrm{~cm}^{-2}$. While smaller than bulk due to a reduction in the phonon mean free paths, the prediction is still much larger than our experimental data, as shown by the curve in Figure 3(a). If the offset between experiment and prediction for the $100 \mathrm{~nm}$ film is due to $h$ at the SiC-AlN interface, its value would need to be $h \sim 10 \mathrm{MW} / \mathrm{m}^{2} \mathrm{~K}$ at $300 \mathrm{~K}$. Values this low have only been observed for highly dissimilar materials. ${ }^{31}$ The TEM image in Figure 3(c), reproduced from Reitmeier et al. ${ }^{18}$ for $100 \mathrm{~nm}$ AlN films grown on $\mathrm{SiC}$ in the same reactor under the same conditions, shows extreme disorder near the interface. The authors conclude that the initial layers of AlN are nonstoichiometric and as a result contain a very high density of point defects and vacancy clusters. This additional disorder is the likely cause of the increased interface resistance (low $h$ ), and is not redundant with the phonon dislocation scattering accounted for by $\tau_{D}$ within the film. In Figure 3(a), the measurements are also compared with the Cahill-Pohl theoretical lower limit to thermal conductivity of disordered crystals. ${ }^{25}$ Agreement of thermal conductivity measurements with the magnitude and trend of the Cahill-Pohl limit is likely coincidental, reflecting only that the highly dislocated AlN film and defective AlN-SiC interface combine to create an apparent disordered behavior.

This research shows that the effective thermal conductivities in the layers of Group III nitride-based LEDs are markedly lower than the bulk thermal conductivities of their constituent materials. These effective values will improve estimates of the MQW operating temperature, used to predict efficiency and lifetime of the LED. Further, this work identifies thermal conductivity as a figure of merit for Group III nitride films used in technologies where heat is a byproduct, e.g., LEDs, HEMTs, and select next generation solar cells. It is apparent that the major source of thermal resistance in the film stack is at the AlN-SiC interface. From a scientific standpoint, a predictive tool for estimating the relaxation time due to phonon dislocation scattering may hold utility for understanding thermal conductivity reductions in other material systems.

The work was supported in part by NSF Grant \#1133394. The authors express their appreciation to Cree, Inc. for providing the $\mathrm{SiC}$ substrates, to the personnel in the CMU clean room and the MEMS laboratories for sample micro-fabrication, and to Alan McGaughey for helpful discussions regarding phonon transport.

\footnotetext{
1"Solid-State Lighting Research and Development, Multi-Year Program Plan FY'09-FY'15," Navigant Consulting, Inc., Radcliff Consulting, Inc., and SSLS Inc. for the Building Technologies Program, EERE, DOE, 2009.

2" Thermal Management of White LEDs," PNNL-SA-51901, Building Technologies Program, EERE, DOE, 2009.

${ }^{3}$ W. L. Liu and A. A. Balandin, Appl. Phys. Lett. 85, 5230 (2004).

${ }^{4}$ G. A. Slack, R. A. Tanzilli, R. O. Pohl, and J. W. Vandersande, J. Phys. Chem. Solids 48, 641 (1987).
}

${ }^{5}$ Y. K. Koh, Y. Cao, D. G. Cahill, and D. Jena, Adv. Funct. Mater. 19, 610 (2009).

${ }^{6}$ M. Dinescu, A. Jacquot, B. Lenoir, A. Dauscher, P. Verardi, F. Craciun, M. Stolzer, and M. Gartner, Appl. Surf. Sci. 186, 507 (2002).

${ }^{7}$ W. L. Liu and A. A. Balandin, J. Appl. Phys. 97, 073710 (2005).

${ }^{8}$ G. J. Riedel, J. W. Pomeroy, K. P. Hilton, J. O. Maclean, D. J. Wallis, M. J. Uren, T. Martin, U. Forsberg, A. Lundskog, A. Kakanakova-Georgieva, G. Pozina, E. Janzen, R. Lossy, R. Pazirandeh, F. Brunner, J. Wurfl, and M. Kuball, IEEE Electron Dev. Lett. 30, 103 (2009).

${ }^{9}$ A. Sarua, H. Ji, K. P. Hilton, D. J. Wallis, M. J. Uren, T. Martin, and M. Kuball, IEEE Trans. Electron Devices 54, 3152 (2007).

${ }^{10}$ A. Manoi, J. W. Pomeroy, N. Killat, and M. Kuball, IEEE Electron Device Lett. 31, 1395 (2010).

${ }^{11}$ M. Asheghi, K. Kurabayashi, R. Kasnavi, and K. E. Goodson, J. Appl. Phys. 91, 5079 (2002).

${ }^{12}$ W. J. Liu and M. Asheghi, J. Heat Transfer-Trans. ASME 128, 75 (2006).

${ }^{13}$ B. A. Danilchenko, T. Paszkiewicz, S. Wolski, A. Jezowski, and T. Plackowski, Appl. Phys. Lett. 89, 061901 (2006).

${ }^{14}$ C. Dames and G. Chen, in Thermoelectrics Handbook: Macro To Nano, edited by D. M. Rowe (CRC, Boca Raton, 2006), p. 1 v. (various pagings).

${ }^{15}$ G. B. Stringfellow, Organometallic Vapor-Phase Epitaxy: Theory and Practice, 2nd ed. (Academic, San Diego, 1999).

${ }^{16}$ Robert F. Davis, Comprehensive semiconductor science and technology (Elsevier, Boston, MA, 2011).

${ }^{17}$ R. F. Davis, S. Einfeldt, E. A. Preble, A. M. Roskowski, Z. J. Reitmeier, and P. Q. Miraglia, Acta Mater. 51, 5961 (2003).

${ }^{18}$ Z. J. Reitmeier, S. Einfeldt, R. F. Davis, X. Y. Zhang, X. L. Fang, and S. Mahajan, Acta Mater. 57, 4001 (2009).

${ }^{19}$ D. G. Cahill, Rev. Sci. Instrum. 61, 802 (1990).

${ }^{20}$ S. M. Lee and D. G. Cahill, J. Appl. Phys. 81, 2590 (1997).

${ }^{21}$ T. Yamane, N. Nagai, S. Katayama, and M. Todoki, J. Appl. Phys. 91, 9772 (2002).

${ }^{22}$ J. H. Kim, A. Feldman, and D. Novotny, J. Appl. Phys. 86, 3959 (1999).

${ }^{23}$ G. Kamler, J. L. Weyher, I. Grzegory, E. Jezierska, and T. Wosinski, J. Cryst. Growth 246, 21 (2002).

${ }^{24}$ C. Mion, J. F. Muth, E. A. Preble, and D. Hanser, Appl. Phys. Lett. 89, 092123 (2006).

${ }^{25}$ D. G. Cahill, S. K. Watson, and R. O. Pohl, Phys. Rev. B 46, 6131 (1992).

${ }^{26}$ B. C. Daly, H. J. Maris, A. V. Nurmikko, M. Kuball, and J. Han, J. Appl. Phys. 92, 3820 (2002).

${ }^{27}$ Y. K. Koh, Ph.D. dissertation, University of Illinois at Urbana-Champaign, 2010.

${ }^{28}$ G. A. Slack, L. J. Schowalter, D. Morelli, and J. A. Freitas, J. Cryst. Growth 246, 287 (2002).

${ }^{29}$ J. Zou, D. Kotchetkov, A. A. Balandin, D. I. Florescu, and F. H. Pollak, J. Appl. Phys. 92, 2534 (2002).

${ }^{30}$ D. Kotchetkov, J. Zou, A. A. Balandin, D. I. Florescu, and F. H. Pollak, Appl. Phys. Lett. 79, 4316 (2001).

${ }^{31}$ H. K. Lyeo and D. G. Cahill, Phys. Rev. B 73, 144301 (2006). 